\title{
Evaluación del uso de un EVS y de mUVies en las aulas: una visión del profesorado y del alumnado
}

\author{
Calvo-Roselló, Vicenta ${ }^{\mathrm{a}}$; López-Rodríguez, $\mathbf{M}^{\mathrm{a}}$ Isabel $^{\mathrm{b}}$; Ruiz-Ponce, Félix ${ }^{\mathrm{c}}$ \\ ${ }^{a}$ Dpto. de Matemática Aplicada. E.T.S. Arquitectura, Universitat Politècnica de València, España, \\ vcalvo@mat.upv.es, 'bpto. de Economía Aplicada. Facultat d'Economia, Universitat de València, \\ España, Maria.I.Lopez@uv.es, 'D Dpto. de Economía Aplicada. Facultat d’Economia, Universitat de \\ València, España, Felix.Ruiz@uv.es
}

\begin{abstract}
Use of the Technologies of Information and Communication (TIC's) and different innovative methodologies, which have raised in a high percentage of the subjects in the current Study Plans, have been a great help in the selflearning of our students. This is the case in one subject of quantitative character in the Degree in Finance and Accounting in the University of Valencia.

In this paper it is exposed, for the case of that subject, the point of view of each of the agents in the teaching-learning process about the use of an "electronic voting system" (EVS), the clickers, and supporting movies. While the first one has been used throughout the class sessions, the second one has been an optional material for the students.

After a first analyse we deduce that, despite it has been a very hard burden for the teaching staff, it has become a satisfying experience. And, this has been not only due to the academic results of the students but also to their very good acceptance. They have valued very positively both, the clickers and the movies.
\end{abstract}

Keywords: TIC's, EVS, clickers, mUVies, Survey, Teaching Innovation, Academic Performance.

\section{Resumen}

La utilización de las Tecnologías de la Información y la Comunicación (TICs) y de las diferentes metodologías docentes innovadoras, que han hecho su aparición en un alto porcentaje de las materias que componen los actuales planes de estudio, ha resultado de gran ayuda en el proceso de autoaprendizaje del alumnado. Este es el caso de una asignatura de carácter 
cuantitativo del grado en Finanzas y Contabilidad de la Universitat de València.

En el trabajo que se presenta, se expone, para el caso de dicha materia, la visión de cada uno de los agentes implicados en el proceso de enseñanzaaprendizaje respecto al uso de un "electronic voting system" (EVS), los clickers, y de vídeos de apoyo (mUVies). Mientras que el primero se ha utilizado a lo largo de las sesiones de la asignatura, el segundo ha constituido un material de uso optativo por parte del alumnado.

Tras un primer análisis se deduce que, aunque para el equipo docente ha resultado una carga de trabajo importante, ésta ha resultado satisfactoria, no sólo por los resultados de la evaluación del alumnado sino por la acogida de éste, que valora muy positivamente el uso tanto de mUVies como de clickers.

Palabras clave: TIC's, EVS, clickers, mUVies, Cuestionario, Innovación Docente, Rendimiento Académico.

\section{Introducción}

En el proyecto de Innovación Docente de la Universitat de València "Elaboración de materiales interactivos y multidisciplinares para favorrecer el aprendizaje y la evaluación en los estudios de grado", apostamos por el uso de un electronic voting system (EVS), los clickers, que han demostrado su eficacia en el aumento de la interacción y la atención en el aula y en la mejora del rendimiento académico del alumnado (Calvo , V., López, M. I. y Ruiz, F. 2017; Caballer, M. y Pardo-García, C. 2014; Barac, M. y Pardo-García, C. 2015) tanto en estudios de grado como en estudios de máster (López, M.I. et al, 2015; López, M.I y Barac, M., 2016).

Por otro lado, el crecimiento, en los últimos años, de los Massive Open Online Courses (MOOC's) muestra el potencial educativo de los videos docentes (Casasús, T.; Ivars, A.; López, M.I. 2018; García Aretio, L. 2017) . Existen multitud de plataformas online para la creación, edición y divulgación de videos (Youtube, Coursera, edX, GoReact, edPuzzle,...). En nuestro caso hemos utilizado el servidor mmedia y el repositorio Roderic de la Universitat de València (UV) así como el canal de Youtube del Servei de Formació Permanent i Innovació Educativa (SFPIE) de la UV, los denominados "mUVies". Una de sus aplicaciones más utilizadas es en el modelo de Flip Teaching o "clase inversa". Como ejemplos de los estudios que analizan su uso se pueden citar, entre otros, los de Pueo, B. et 
al. (2014), Gross, D, et alt. (2015) o Singh, V. et alt. (2016). En Stoltzfus, J. R. and Libarkin, J. (2016), incluso se discute la influencia de la forma del aula en la mejora del aprendizaje del alumnado.

Las nuevas tecnologías ayudan en gran medida al aprendizaje autónomo, pero es imprescindible decir que no hay atajos en el aprendizaje. No es eso lo que se pretende con los vídeos de apoyo a la docencia. La adquisición de conocimientos necesita de un tiempo de asimilación ineludible. Por eso, cuando los vídeos incluyen pausas pautadas para que el alumno pueda practicar, aunque sea mínimamente, lo que se le está explicando, funcionan mucho mejor. Aunque, a título personal, pensamos que, en ningún caso, sustituyen la labor del profesor en el aula. El contacto directo, la interacción que se produce en el aula son insustituibles. Sin embargo, en esta era de las TIC's, los videos pueden ser una herramienta muy útil para motivar al alumnado y complementar la docencia más tradicional.

Como es una herramienta optativa, que el alumno tiene a su disposición en cualquier momento y utiliza cuando quiere, debe ser suficientemente atractivo e interesante, porque en otro caso, sencillamente, no lo utilizará. Para que sea realmente útil un video docente debe cumplir unas condiciones básicas, entre las que se pueden citar: a) ser breve, conciso y directo, b) ser entretenido y dinámico, c) contener pausas pautadas.d) utilizar unlenguaje claro y directo, sin circunloquios innecesarios.

Con estas bases se ha trabajado y, como se verá a continuación, la respuesta del alumnado, al uso combinado de clickers y videos, ha sido excelente.El estudio se realiza teniendo en cuenta las respuestas de los estudiantes de Estadística del grado en Finanzas y Contabilidad de la UV a un cuestionario ad-hoc que se confeccionó, buscando conocer la percepción que el alumnado tuvo de dicho uso. La estructura del cusetionario fue las siguiente: cuestiones con respuesta escala Likert (de 5 ítems) y una de la valoración (en una escala de 0 a 10) del uso para cada una de las herramientas y una pregunta abierta acerca del $\mathrm{n}^{\circ}$ de visualizaciones que el alumno había necesitado realizar hasta comprender el contenido del vídeo (o mUVie).

\section{Metodología}

La información empírica utilizada para la elaboración del trabajo planteado se ha obtenido haciendo uso de un muestreo casual (Latorre et al, 2003) mUViesRespecto a la metodología considerada, en la que se combinan técnicas descriptivas e inferenciales, se han seleccionado tanto las representaciones gráficas como las medidas de reducción más adecuadas al estudio. Ssu uso permitirá, tras la realización del análisis exploratorio pertinente, obtener no sólo el volumen de trabajo que ha supuesto para el alumnado la 
utilización de las herramientas propuestas sino además conocer la percepción que el mismo tuvo de su uso a lo largo de su proceso de aprendizaje.

Un proceso inferencial posterior posibilitará acotar, inferior y superiomente, la valoración que el alumnado del grado proporcionaría tanto a los clickers como a los mUVies.

Por último, mediante un análisis comparativo se comprobará, si existe o no algún tipo de diferencia en la percepción del alumnado respecto a las dos herramientas utilizadas. En el caso de que ésta se detectara, se propone como futura línea de investigación el análisis de la significatividad de la misma. Para ello se haría uso de un análisis de datos apareados, que requeriría la comprobación del cumplimiento de la hipótesis de normalidad de la variable d (=diferencia entre las valoraciones dadas a clickers y mUVies), proponiéndose para ello la utilización del test de Kolmogorov-Smirnov.

\section{Resultados}

En cuanto al volumen de trabajo que, para el equipo docente de la asignatura, supuso la puesta en marcha de las TICs (tabla 1), atendiendo a cada herramienta, se ha estimado:

- Uso del EVS: el estudio del manual del software Turningpoint se puede cuantificar en unas 50 horas aproximadamente y la realización de cada sondeo de clickers necesitó unas 5 horas de preparación, habiéndose realizado 3 sondeos. Han sido necesarias, por tanto, 65 horas de trabajo del docente para poner en marcha esta herramienta.

- MUVies: en este caso hay que distinguir dos tareas, a saber, la elaboración del material necesario para la grabación y el tiempo dedicado a la misma. La dedicación estimada, para cada una de ellas, es de 2 horas. Si se tiene en cuenta que se elaboraron 25 mUVies, se obtiene que el docente dedicó unas 100 horas a la realización de este material audiovisual.

Tabla 1. Cómputo del volumen de trabajo del profesorado

\begin{tabular}{|c|c|c|c|c|}
\hline & \multicolumn{2}{|c|}{ CLICKERS } & \multicolumn{2}{|c|}{ VÍDEOS } \\
\hline & $\begin{array}{c}\text { Conocimiento } \\
\text { adecuado HRA } \\
50 \mathrm{~h}\end{array}$ & $\begin{array}{l}\text { Preparación/ } \\
\text { sondeo } \\
5 \mathrm{~h}\end{array}$ & $\begin{array}{c}\text { Preparación } \\
\text { material/vídeo } \\
\text { 2h }\end{array}$ & $\begin{array}{c}\text { Grabación/vídeo } \\
2 \mathrm{~h}\end{array}$ \\
\hline $\mathrm{N}^{\mathrm{o}}$ & & 3 & 25 & 25 \\
\hline SUBTOTAL & $50 \mathrm{~h}$ & $15 \mathrm{~h}$ & $50 \mathrm{~h}$ & $50 \mathrm{~h}$ \\
\hline TOTAL & \multicolumn{2}{|c|}{$65 \mathrm{~h}$} & \multicolumn{2}{|c|}{$100 \mathrm{~h}$} \\
\hline
\end{tabular}

Fuente: Elaboración propia

Obsérvese que el volumen de trabajo total se cifra en unas 165 horas, o lo que es equivalente, en cerca de 21 días laborales (de $8 \mathrm{~h}$.) completos. 
Respecto a la dedicación del alumnado, es decir, el tiempo que dedicó al uso de las herramientas consideradas, en la tabla 2 se recogen los datos y el resultado de las operaciones que permiten concluir que cada alumno dedicó 1.5 horas al uso de los clickers (3 sondeos de media hora de duración cada uno de ellos) y de 9.5 horas, por término medio, a la visualización de vídeos. Esto es, un total de 11 horas o, equivalentemente, de aproximadamente 1.5 días laborables (de $8 \mathrm{~h}$.).

Tabla 2. Cómputo de la dedicación del alumnado

\begin{tabular}{|c|c|c|c|}
\hline & \multirow{2}{*}{$\begin{array}{r}\text { CLICKERS } \\
\text { Realización/sondeo } \\
0.5 \mathrm{~h}\end{array}$} & \multicolumn{2}{|c|}{ VÍDEOS } \\
\hline & & $\begin{array}{c}\mathbf{N}^{\mathbf{o}} \text { visualizaciones/video } \\
1.52\end{array}$ & $\begin{array}{c}\text { Tiempo/vídeo } \\
15\end{array}$ \\
\hline $\mathrm{N}^{\mathrm{o}}$ & 3 & 25 & \\
\hline SUBTOTAL & $1.5 \mathrm{~h}$ & 38 visualizaciones & $570^{\prime} \cong 9.5 \mathrm{~h}$ \\
\hline TOTAL & $1.5 \mathrm{~h}$ & 9. & \\
\hline
\end{tabular}

Hay que tener en cuenta que en el cuestionario ad-hoc se incluyó una pregunta que permitió obtener que el $\mathrm{n}^{\mathrm{o}}$ de visualizaciones que, por término medio, fueron necesarias para la comprensión del contenido del video fue de 1.52. Como la duración media de los mismos era de 15 minutos y el $\mathrm{n}^{\mathrm{o}}$ de vídeos de 25 , el tiempo medio de dedicación de un alumno, que ha visualizado todos los vídeos, es de 570 minutos $(9.5 \mathrm{~h})$.

Con la finalidad de realizar un estudio comparativo entre ambas herramientas, se consideran las preguntas coincidentes: 2 escala Likert (C1: ¿El uso de los clickers/vídeos te ha ayudado a comprender mejor los conceptos de la asignatura? y C2: ¿El uso de los clickers/vídeos te ha ayudado a preparar mejor el examen final?) y una pregunta de entrada numérica múltiple (valoración del uso de clickers/vídeos en escala de 0 a 10).

Las tablas 3 y 4 , recogen los porcentajes correspondientes a cada uno de los ítems

Tabla 3. Respuestas (escala likert) a Cuestión 1, según herramienta

\begin{tabular}{ccc}
\hline \multicolumn{3}{c}{ ¿Te ha ayudado a comprender mejor los conceptos de la asignatura? } \\
\cline { 2 - 3 } No, en absoluto & clickers & Vídeos \\
\cline { 2 - 3 } Poco & $0 \%$ & $0 \%$ \\
Indiferente & $8.70 \%$ & $4.76 \%$ \\
Bastante & $78.26 \%$ & $0.00 \%$ \\
Mucho & $13.04 \%$ & $57.14 \%$ \\
\hline
\end{tabular}


Tabla 4. Respuestas (escala likert) a Cuestión 2, según herramienta

\begin{tabular}{ccc}
\hline \multicolumn{3}{c}{ ¿Te ha ayudado a preparar el examen final? } \\
\cline { 2 - 3 } No, en absoluto & clickers & Vídeos \\
Poco & $4.35 \%$ & $4.76 \%$ \\
Indiferente & $8.70 \%$ & $4.76 \%$ \\
Bastante & $26.09 \%$ & $9.52 \%$ \\
Mucho & $43.48 \%$ & $42.86 \%$ \\
\hline
\end{tabular}

Fuente: Elaboración propia

A partir de la Tabla 3 se puede concluir que el porcentaje de estudiantes que seleccionaron la opción "Bastante" fue superior al 50\% para ambas herramientas. Si además se agregan los casos más favorables (Bastante y Mucho) se obtiene que más del $90 \%$ del alumnado percibió que el uso tanto de clickers como de mUVies les permitió comprender mejor los conceptos de la asignatura. Concretamente ese porcentaje se elevó al 91.3\% para el caso de los clickers y al $95.24 \%$ para mUVies.

En el caso de la cuestión C2 (tabla 4), aunque los resultados son ligeramente "inferiores", no deja de sorprender que el porcentaje de estudiantes que percibieron que les había ayudado bastante a preparar el examen final fue superior al $42 \%$, pero si además se consideran los que percibían que les había ayudado mucho, dichos porcentajes se elevan notablemente, pasando a ser del $60.87 \%$ para clickers y del $80.96 \%$ para mUVies.

La figura 1 permite visualizar lo indicado:

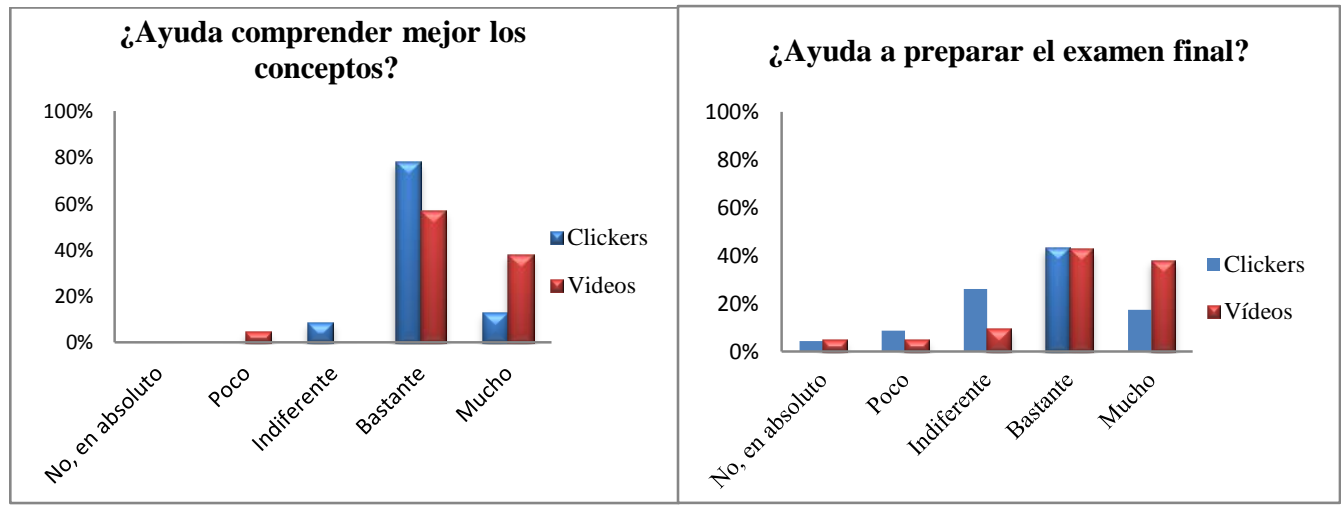

a)

b)

Fig. 1 Comparativa respuestas a) Cuestión 1; b) Cuestión 2

Centrando la comparativa en la valoración proporcionada a su uso, a partir de las medidas de reducción más relevantes contenidas en la tabla 5 , 
Tabla 5. Medidas de posición de la valoración ( 0 a 10), según herramienta

\begin{tabular}{ccc}
\hline \multicolumn{3}{c}{ Valoración uso (escala de 0 a 10) } \\
\hline Valoración media & clickers & Vídeos \\
\cline { 2 - 3 } Coeficiente de variación de Pearson & 8.04 & 8.57 \\
Mínimo & 0.2 & 0.18 \\
Máximo & 2 & 4 \\
& 10 & 10 \\
\hline
\end{tabular}

se observa una mayor puntuación media para los vídeos ( 8.57 frente a 8.04$)$, con menor dispersion, atendiendo al valor del coeficiente de variación de Pearson ( 0.18 para vídeos y 0.2 para clickers).

Sorprende, gratamente, que la puntuación minima no sea 0 en ninguno de los dos casos, mientras que la máxima sí alcanza el mayor valor que podia otorgarse (tanto a clickers como a vídeos).

Además, atendiendo a los datos de la Tabla 6, que recoge los porcentajes de alumnos que adjudicaron una puntuación superior o igual a 7, a 8 y a 9 a ambas herramientas

Tabla 6. Porcentaje de valoraciones altas, según herramienta

\begin{tabular}{lcc}
\hline \multicolumn{3}{c}{ Valoración uso (escala de 0 a 10) } \\
\hline & clickers & Vídeos \\
\cline { 2 - 3 } Valoración superior o igual a 7 & $91.30 \%$ & $95.24 \%$ \\
Valoración superior o igual a 8 & $78.26 \%$ & $80.95 \%$ \\
Valoración superior o igual a 9 & $39.13 \%$ & $52.38 \%$ \\
\hline
\end{tabular}

se observa que el porcentaje de alumnos que le asignaron alta puntuación a ambas herramientas fue elevado y siempre mayor en vídeos que en clickers. Por ejemplo, más de un $90 \%$ los calificaron con una nota superior a 7 (el $91.3 \%$ en el caso de clickers y el $95.24 \%$ en el de vídeos) y si se considera como cota inferior el 9 se observa que el $39.13 \%$ del alumnado asignó un 9 mínimo al uso de los clickers y más del 50\% al uso de los vídeos.

Dados los buenos resultados, surge la siguiente pregunta: ¿serían extrapolables las conclusiones a todo el alumnado?. Para dar respuesta a la misma se realiza una análisis inferencial mediante el uso de herramientas básicas (obtención del sesgo y curtosis estandarizados y el gráfico de probabilidad Normal). Como paso previo se utiliza el diagrama de Box-Whisker que permite detectar, en caso de que existan, datos anómalos. La figura 2 contiene dicho diagrama para las valoraciones de clickers y de mUVies. 


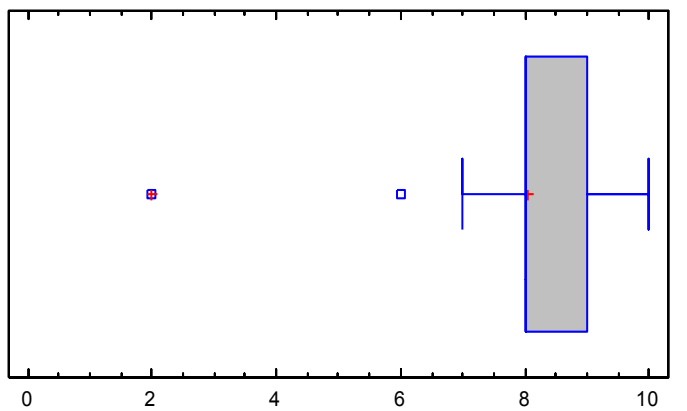

a)

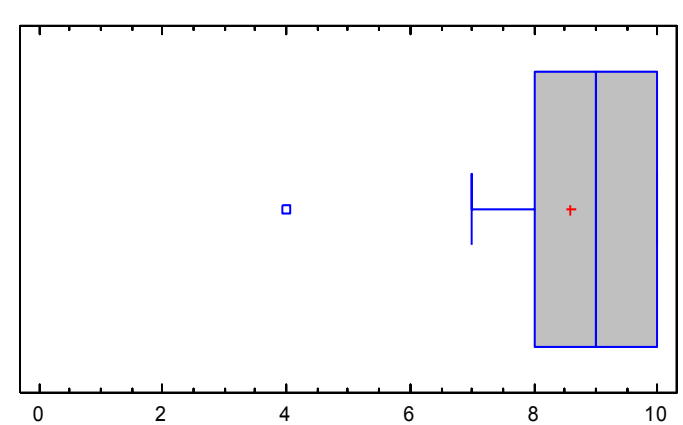

b)

Fig. 2 Diagrama de Box-Whisker para valoración de a) clickers; b) Videos

De su observación se deduce que hay dos datos anómalos en el caso de la valoración de clickers y uno en el de vídeos. Datos que se procede a eliminar.

Las medidas de posición, tras la eliminación de dichos datos, contenidas en la tabla 7

Tabla 7. Medidas de posición de la valoración (0 a 10), según herramienta

\begin{tabular}{|c|c|c|}
\hline \multicolumn{3}{|c|}{ Valoración uso (escala de 0 a 10) tres eliminación datos anómalos } \\
\hline & clickers & Vídeos \\
\hline Valoración media & 8.43 & 8,8 \\
\hline Desviación típica & 0.93 & 1,15 \\
\hline Coeficiente de variación de Pearson & 0.11 & 0.13 \\
\hline Mínimo & 7 & 7 \\
\hline Máximo & 10 & 10 \\
\hline Sesgo Estandarizado & 0.43 & -0.48 \\
\hline Curtosis Estandarizada & -0.55 & -1.34 \\
\hline
\end{tabular}

se concluye que se mantienen altas calificaciones medias y superiores para el uso de vídeos. Observando el valor del sesgo estandarizado y la curtosis estandarizada (entre -2 y 2), se puede asumir que los datos con los que se trabaja provienen de una población Normal.

Dicha afirmación es sustentada por los gráficos de probabilidad normal (fig. 3), al no encontrarse los datos " muy alejados de la recta". 


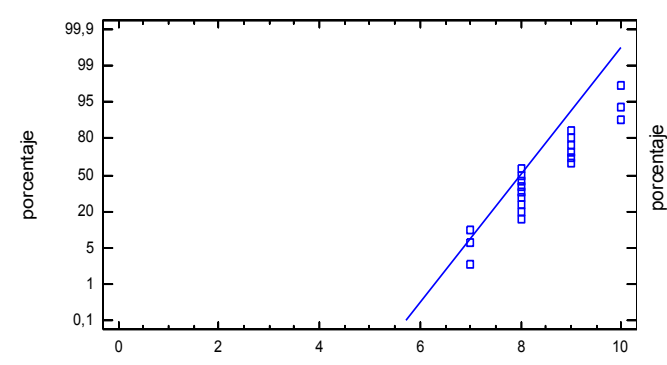

a)

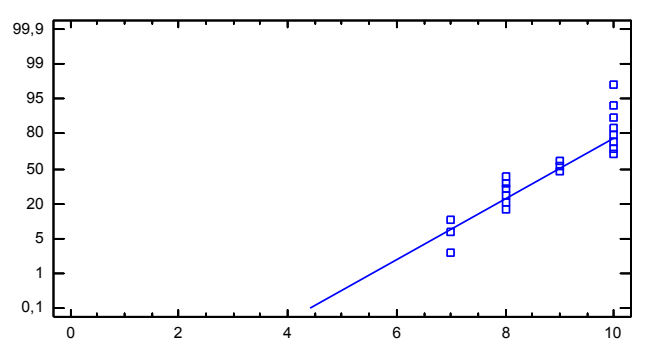

b)

Fig. 3 Gráfico de Probabilidad Normal para valoración de a) clickers; b) Videos

Al poderse asumir la Normalidad para la valoración proporcionada a ambas herramientas, son de aplicación las propiedades de dicho modelo. Una de ellas enuncia que el $99.73 \%$ de los datos de una Normal se encuentran en el intervalo centrado en la media y con amplitud $6 \sigma$. Así, tomando como estimadores de la media y la varianza poblacional los mismos parámetros muestrales y atendiendo a los valores de los mismos contenidos en la tabla 7 se concluye que en $99.73 \%$ de los casos los alumnos valorarán el uso de los clickers con una nota mínima de $6.64(=\mu-3 \sigma=8.43-3 \cdot 0.93)$ y máxima de $10(=$ mín $(10,11.22=\mu+3 \sigma=$ $8.43+3 \cdot 0.93))$. En el caso de los vídeos las puntuaciones mínima y máxima serían de 5.35 $(=\mu-3 \sigma=8.8-3 \cdot 1.15)$ y de $10(=\operatorname{mín}(10,12.25=\mu+3 \sigma=8.8+3 \cdot 1.15))$

\section{Conclusiones}

Algunas de las conclusiones más relevantes que se deducen del estudio son las siguientes:

- El alumnado ha acogido muy positivamente el uso de estas dos herramientas, tanto los clickers como los mUVies. En efecto, las respuestas reflejan un alto grado de satisfación del alumnado, pues en ningún caso el porcentaje de alumnos que indicaban estar "bastante o muy satisfechos" fue inferior al $60 \%$ y en la mayoría de los casos superaba el $80 \%$.

- El porcentaje de alumnos que le asignaron alta puntuación a ambas herramientas fue elevado y siempre mayor en mUVies que en clickers.

- La puesta en marcha de TIC ha supuesto una carga de trabajo importante para el equipo docente de la asignatura. Ha sido mayor en el caso de los mUVies, con una estimación de unas $100 \mathrm{~h}$ de trabajo, que en el de los clickers, con unas $65 \mathrm{~h}$ de dedicación.

- Respecto a la dedicación del alumnado, también fue mayor, como es lógico, en el caso de los videos, unas $9.5 \mathrm{~h}$, frente al tiempo dedicado a los clickers, $1.5 \mathrm{~h}$.

- Esta dedicación ha sido provechosa puesto que los estudiantes han percibido una mejora de su rendimiento en la asignatura. 
Por tanto, y a modo de conclusión global, aunque la incorporación de las herramientas ha supuesto no sólo la implicación del profesorado y del alumnado sino un incremento elevado en el volumen de trabajo (15 veces superior para el docente), los resultados son muy satisfactorios y conducen a valorar muy positivamente la utilización tanto de clickers como de mUVies.

\section{Agradecimientos}

Expresamos nuestro agradecimiento al Servei de Formació Permanent i Innovació Educativa así como a los Vicerrectorats d'Estudis de Grau i Política Lingüística i de Polítiques de Formació i Qualitat Educativa de la Universitat de València por la concesión del proyecto de innovación educativa UV-SFPIE_GER17-585541 que ha financiado parcialmente esta comunicación.

\section{Referencias}

Barac, M. y Pardo-García, C. (2015). "Uso de los clickers en asignaturas con características docentes diferentes: motivación y evaluación del alumnado". En IV jornades IDES. Valencia. Disponible en <http://www.uv.es/econdocs/ides2015/ Llibre_actes_IV_jornades_IDES_2015>

CaballerTarazona, M. y Pardo-García, C. (2014). "Statistics continuous assessment through an activity using an interactive voting system" en Edulearn 14. Barcelona. IATED, pp. 2939-2944.

Calvo Roselló, V.; López Rodríguez, M.I.; Ruiz Ponce, F.. (2017). "Uso de un Electronic Voting System: una radiografía del aula universitaria en tiempo real". INNODOCT/17. Valencia. Pendiente de publicación.

Casasús, T.; Ivars,A.; López M.I. (2018): Present and future of the e-learning in economics schools and faculties. Multidisciplinary Journal for Education, Social and Technological Sciences, Vol. $5 \mathrm{~N}^{\mathrm{o}} 1$ (2018): pp. 44-64

García Aretio, L. (2017). Los MOOC están muy vivos. REspuestas a algunas preguntas. Revista Iberoamericana de Educación a Distancia, 20(1), 9-27.

Gross, D.; Pietri, E. S.; Anderson, G.; Moyano-Camihort, K.; Graham, M. J. (2015). Increased preclass preparation underlies student outcome improvement in the flipped classroom. CBE Life Sciences Education, 14(4), 1-8. doi:http://doi.org/10.1187/cbe.1502-0040

Latorre, A.; Del Rincón, D.; Arnal, J. (2003). Bases metodológicas de la investigación educativa. Barcelona: Ediciones Experiencia. 
López Rodríguez, M.I.; Palací López, J.;Palací López, D. (2015). "Use of ICTs in degree studies: a descriptive analysis”. En Sevilla. ICERI 15. IATED, pp. 2286-2290.

López, M.I y Barac, M. (2016). "Uso de los clickers en una asignatura de diseño de experimentos". En Valencia. V jornades IDES .

Pueo, B.; Jimenez-Olmedo, J. M.; Penichet-Tomas, A., Carbonell-Martinez, J. A. (2017). Aplicación de la herramienta EDpuzzle en entornos de aprendizaje individuales dentro del aula. En: Roig-Vila, Rosabel (ed.). Investigación en docencia universitaria. Diseñando el futuro a partir de la innovación educativa. Barcelona: Octaedro, 2017. ISBN 978-84-9921-935-6, pp. 694-702

Singh, V.; Abdellahi, S.; Maher, M. Lou;Latulipe, C. (2016). The video collaboratory as a learning environment. In Proceedings of the 47th ACM Technical Symposium on Computer Science Education (SIGCSE '16) (pp. 352-357). doi:http://doi.org/10.1145/2839509.2844588

Stoltzfus, J. R. ; Libarkin, J. (2016). Does the room matter? Active learning in traditional and enhanced lecture spaces. CBE Life Sciences Education, 15(4), 1-10. doi:http://doi.org/10.1187/cbe.16-03-0126 